\title{
DIMENSION INEQUALITIES OF MULTIFRACTAL HAUSDORFF MEASURES AND MULTIFRACTAL PACKING MEASURES
}

\author{
L. OLSEN
}

\begin{abstract}
Let $\mu$ be a Borel probability measure on $\mathrm{R}^{d}$. We study the Hausdorff dimension and the packing dimension of the multifractal Hausdorff measure $\mathscr{H}_{\mu}^{q, t}$ and the multifractal packing measure $\mathscr{P}_{\mu}^{q, t}$ introduced in [L. Olsen, A multifractal formalism, Advances in Mathematics 116 (1995), 82-196]. Let $b_{\mu}$ denote the multifractal Hausdorff dimension function and let $B_{\mu}$ denote the multifractal packing dimension function introduced in [Olsen, op cit]. For a fixed $q \in \mathrm{R}$, we obtain bounds for the Hausdorff dimension and the packing dimension of $\mathscr{H}_{\mu}^{q, b_{\mu}(q)}$ and $\mathscr{P}_{\mu}^{q, B_{\mu}(q)}$ in terms of the subdifferential of $b_{\mu}$ and $B_{\mu}$ at $q$. For $q=1$, our result reduces to
\end{abstract}

(*) $\quad-D_{+} B_{\mu}(1) \leq \liminf _{r \searrow 0} \frac{\log \mu B(x, r)}{\log r} \leq \limsup _{r \searrow 0} \frac{\log \mu B(x, r)}{\log r} \leq-D_{-} B_{\mu}(1) \quad$ for $\mu$-a.a. $x$

where $D_{-} B_{\mu}(1)$ and $D_{+} B_{\mu}(1)$ denote the left and right derivative of $B_{\mu}$ at 1 . Inequality $(*)$ improves a similar result obtained independently by Y. Heurteaux and S.-Z. Ngai. It follows from (*) that if the mulifractal box dimension spectrum (or $L^{q}$ spectrum) $\tau_{\mu}$ of $\mu$ is differentiable at 1 then $-\tau_{\mu}^{\prime}(1)$ equals the entropy dimension (or information dimension) of $\mu$. This result has been conjectured in the physics literature and proved rigorously in certain special cases.

\section{Contents}

1. Introduction.

2. Statement of Results.

3. Multifractal Density Theorems.

4. Proofs of the Main Results. References

\section{Introduction}

Let $\mu$ is a probability measure on a metric space $X$. The Hausdorff multifractal spectrum function, $f_{\mu}$, and the packing multifractal spectrum function, $F_{\mu}$, of $\mu$ are defined by

Received August 2, 1997. 


$$
f_{\mu}(\alpha)=\operatorname{dim}\left\{x \in X \mid \lim _{r \searrow 0} \frac{\log \mu B(x, r)}{\log r}=\alpha\right\}, \quad \alpha \geq 0,
$$

and

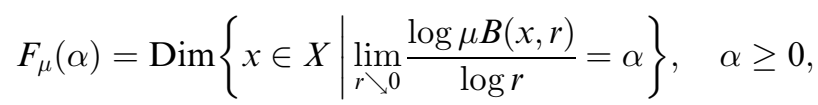

respectively, where $B(x, r)$ denotes the closed ball with center $x$ and radius $r$, dim denotes the Hausdorff dimension and Dim denotes the packing dimension. It has recently been conjectured in the physics literature [HJKPS,HP] that for "good" measures the following result, known as the Multifractal Formalism, holds.

The Multifractal Formalism - A Physics Folklore Theorem

(i) For each $q \in \mathrm{R}$ there exists a unique number $\tau(q)$ such that

$$
\lim _{r \searrow 0} \sup \sum_{i} \mu\left(B\left(x_{i}, r_{i}\right)\right)^{q}\left(2 r_{i}\right)^{t}=\left\{\begin{array}{cl}
\infty & \text { if } t<\tau(q) \\
0 & \text { if } \tau(q)<t
\end{array},\right.
$$

where the supremum is taken over all countable packings $\left(B\left(x_{i}, r_{i}\right)\right)_{i}$ of the support of $\mu$ with $\sup _{i} r_{i} \leq r$.

(ii) For each $q \in \mathrm{R}$ the following limit exists and equals $\tau(q)$,

$$
\tau(q)=\lim _{r \searrow 0} \frac{\log \left(\sup \sum_{i} \mu\left(B\left(x_{i}, r\right)\right)^{q}\right)}{-\log r}
$$

where the supremum is taken over all countable packings $\left(B\left(x_{i}, r\right)\right)_{i}$ of the support of $\mu$.

(iii) The function $\tau$ is convex, decreasing and differentiable with $\tau(1)=0$. Furthermore, if we let

$$
\alpha(q)=-\tau^{\prime}(q)
$$

then

$$
f_{\mu}(\alpha(q))=q \alpha(q)+\tau(q) \text { for } q \in \mathrm{R},
$$

or equivalently

$$
f_{\mu}(\alpha)=\tau^{*}(\alpha) \text { for } \alpha \in \mathbf{R},
$$

where $\tau^{*}$ denotes the Legendre transform of $\tau$, i.e. $\tau^{*}(\alpha)=\inf _{q}(\alpha q+\tau(q))$.

Very recently there has been an enormous interest in verifying the Multifractal Formalism and computing the multifratcal spectrum of measures in the mathematical literature, and within the last 3 or 4 years the multifractal spectra of various classes of measures in Euclidean space $\mathrm{R}^{d}$ exhibiting some 
degree of self-similarity have been computed rigorously. Specifically, the Multifractal Formalism (1.3) has been proved rigorously for random and non-random self-similar measures [AP, CM, EM, O11, O12, RM], for selfconformal measures $[\mathrm{KG}, \mathrm{Pa}, \mathrm{PW}, \mathrm{Ra}]$ and for self-affine measures $[\mathrm{Ki}$, O13].

The proofs of the Multifractal Formalism (1.3) in the above-mentioned references [AP, CM, EM, KG, Ki, O11, O12, O13, Pa, PW, Ra, RM] are all based on the same key idea. The upper bound for $f_{\mu}(\alpha(q))$, i.e. the inequality $f_{\mu}(\alpha(q)) \leq q \alpha(q)+\tau(q)$, is obtained by a standard covering argument (involving Besicovitch's Covering Theorem or Vitali's Covering Theorem). The lower bound for $f_{\mu}(\alpha(q))$, i.e. the inequality $f_{\mu}(\alpha(q)) \geq q \alpha(q)+\tau(q)$, is usually much harder to prove and is obtained as follows. Fix $q \in \mathrm{R}$. By making use of the ergodic theorem and a number of ad hoc arguments based on the particular setting, the authors construct a "natural" probability measure $\mu_{q}$ with the following two properties (for positive functions $f, g:(0, \infty) \rightarrow(0, \infty)$, we will write $f(r) \asymp g(r)$ as $r \searrow 0$ if $\lim _{r \searrow 0} \frac{\log f(r)}{\log r}=$ $\left.\lim _{r \searrow 0} \frac{\log g(r)}{\log r}\right)$ :

The support condition. The measure $\mu_{q}$ is tailored to see only the multifractal decomposition set $\left\{x \in \mathbf{R}^{d} \mid \lim _{r \searrow 0} \frac{\log \mu B(x, r)}{\log r}=\alpha(q)\right\}$, i.e.

$$
\mu(B(x, r)) \asymp r^{\alpha(q)} \text { as } r \searrow 0 \text { for } \mu_{q} \text {-a.a. } x,
$$

or equivalently

$$
\lim _{r \searrow 0} \frac{\log \mu B(x, r)}{\log r}=\alpha(q) \text { for } \mu_{q} \text {-a.a. } x \text {. }
$$

The local scaling condition. The measure $\mu_{q}$ has the "correct" almost sure local scaling behaviour, i.e.

$$
\mu_{q}(B(x, r)) \asymp \mu(B(x, r))^{q}(2 r)^{\tau(q)} \text { as } r \searrow 0 \text { for } \mu_{q^{-} \text {a.a. } x},
$$

or equivalently (by (1.4))

$$
\lim _{r \searrow 0} \frac{\log \mu_{q} B(x, r)}{\log r}=q \alpha(q)+\tau(q) \text { for } \mu_{q} \text {-a.a. } x .
$$

It is well-known that (1.5) implies that (cf. [Ma, Theorem 6.9])

$$
\inf \left\{\operatorname{dim} E \mid E \subseteq \mathrm{R}^{d} \text { and } \mu_{q}\left(\mathrm{R}^{d} \backslash E\right)=0\right\}=q \alpha(q)+\tau(q)
$$

The inequality $f_{\mu}(\alpha(q)) \geq q \alpha(q)+\tau(q)$ is now easily obtained from (1.4) and (1.6) as follows 


$$
\begin{aligned}
f_{\mu}(\alpha(q)) & =\operatorname{dim}\left\{x \in \mathrm{R}^{d} \mid \lim _{r \searrow 0} \frac{\log \mu B(x, r)}{\log r}=\alpha(q)\right\} & & \\
& \geq \inf \left\{\operatorname{dim} E \mid E \subseteq \mathrm{R}^{d} \text { and } \mu_{q}\left(\mathrm{R}^{d} \backslash E\right)=0\right\} & & \text { [by (1.4)] } \\
& =q \alpha(q)+\tau(q) . & & \text { [by (1.6)] }
\end{aligned}
$$

In an attempt to develop a general theoretical framework for studying the multifractal structure of arbitrary measures, Olsen [O11], Pesin [Pes] and Peyriére [Pey] suggested various ways of defining measures analogous to $\mu_{q}$ in very general settings. For an arbitrary Borel probability measure $\mu$ on a metric space $X$, they introduced two two-parameter families of measures,

$$
\left\{\mathscr{H}_{\mu}^{q, t} \mid q, t \in \mathrm{R}\right\} \quad \text { and } \quad\left\{\mathscr{P}_{\mu}^{q, t} \mid q, t \in \mathrm{R}\right\},
$$

based on certain generalizations of the Hausdorff measure and of the packing measure. Let $E \subseteq X$ and $\delta>0$. A countable family $\mathscr{B}=\left(B\left(x_{i}, r_{i}\right)\right)_{i}$ of closed balls in $X$ is called a centered $\delta$-covering of $E$ if $E \subseteq \cup_{i} B\left(x_{i}, r_{i}\right)$, $x_{i} \in E$ and $0<r_{i}<\delta$ for all $i$. The family $\mathscr{B}$ is called a centered $\delta$-packing of $E$ if $x_{i} \in E, 0<r_{i}<\delta$ and $B\left(x_{i}, r_{i}\right) \cap B\left(x_{j}, r_{j}\right)=\varnothing$ for all $i \neq j$. For $E \subseteq X$, $q, t \in \mathrm{R}$ and $\delta>0$ write

$$
\begin{aligned}
& \overline{\mathscr{H}}_{\mu, \delta}^{q, t}(E)= \inf \left\{\sum_{i} \mu\left(B\left(x_{i}, r_{i}\right)\right)^{q}\left(2 r_{i}\right)^{t} \mid\right. \\
&\left.\left(B\left(x_{i}, r_{i}\right)\right)_{i} \text { is a centered } \delta \text {-covering of } E\right\}, \quad E \neq \varnothing \\
& \overline{\mathscr{H}}_{\mu, \delta}^{q, t}(\varnothing)= 0 \\
& \frac{\mathscr{H}_{\mu}^{q, t}(E)=}{\sup _{\delta>0}} \overline{\mathscr{H}}_{\mu, \delta}^{q, t}(E) \\
& \mathscr{H}_{\mu}^{q, t}(E)=\sup _{F \subseteq E} \overline{\mathscr{H}}_{\mu}^{q, t}(F)
\end{aligned}
$$

and

$$
\begin{aligned}
\overline{\mathscr{P}}_{\mu, \delta}^{q, t}(E)= & \sup \left\{\sum_{i} \mu\left(B\left(x_{i}, r_{i}\right)\right)^{q}\left(2 r_{i}\right)^{t} \mid\right. \\
& \left.\left(B\left(x_{i}, r_{i}\right)\right)_{i} \text { is a centered } \delta \text {-packing of } E\right\}, \quad E \neq \varnothing \\
\overline{\mathscr{P}}_{\mu, \delta}^{q, t}(\varnothing)= & 0 \\
\overline{\mathscr{P}}_{\mu}^{q, t}(E)= & \inf _{\delta>0} \overline{\mathscr{P}}_{\mu, \delta}^{q, t}(E) \\
\mathscr{P}_{\mu}^{q, t}(E)= & \inf _{E \subseteq \cup_{i} E_{i}} \sum_{i} \overline{\mathscr{P}}_{\mu}^{q, t}\left(E_{i}\right) .
\end{aligned}
$$

It follows from [O11] that $\mathscr{H}_{\mu}^{q, t}$ and $\mathscr{P}_{\mu}^{q, t}$ are measures on the family of Borel 
subsets of $X$. The measure $\mathscr{H}_{\mu}^{q, t}$ is of course a multifractal generalisation of the centered Hausdorff measure, whereas $\mathscr{P}_{\mu}^{q, t}$ is a multifractal generalisation of the packing measure. In fact, it is easily seen that the follwing holds for $t \geq 0$,

$$
2^{-t} \mathscr{H}_{\mu}^{0, t} \leq \mathscr{H}^{t} \leq \mathscr{H}_{\mu}^{0, t}, \mathscr{P}^{t}=\mathscr{P}_{\mu}^{0, t}, \overline{\mathscr{P}}^{t}=\overline{\mathscr{P}}_{\mu}^{0, t},
$$

where $\mathscr{H}^{t}$ denotes the $t$-dimensional Hausdorff measure, $\mathscr{P}^{t}$ denotes the $t$ dimensional packing measure and $\overline{\mathscr{P}}^{t}$ denotes the $t$-dimensional prepacking measure. It is easily seen that the measures $\mathscr{H}_{\mu}^{q, t}$ and $\mathscr{P}_{\mu}^{q, t}$, and the pre-measure $\overline{\mathscr{P}}_{\mu}^{q, t}$ in the usual assign way a dimension to each subset $E$ of $X$ (cf. [011]): there exist unique numbers $\Delta_{\mu}^{q}(E), \operatorname{Dim}_{\mu}^{q}(E), \operatorname{dim}_{\mu}^{q}(E) \in[-\infty, \infty]$ such that

$$
\begin{aligned}
& \overline{\mathscr{P}}_{\mu}^{q, t}(E)= \begin{cases}\infty & \text { for } t<\Delta_{\mu}^{q}(E) \\
0 & \text { for } \Delta_{\mu}^{q}(E)<t\end{cases} \\
& \mathscr{P}_{\mu}^{q, t}(E)= \begin{cases}\infty & \text { for } t<\operatorname{Dim}_{\mu}^{q}(E) \\
0 & \text { for } \operatorname{Dim}_{\mu}^{q}(E)<t\end{cases} \\
& \mathscr{H}_{\mu}^{q, t}(E)= \begin{cases}\infty & \text { for } t<\operatorname{dim}_{\mu}^{q}(E) \\
0 & \text { for } \operatorname{dim}_{\mu}^{q}(E)<t\end{cases}
\end{aligned}
$$

The number $\operatorname{dim}_{\mu}^{q}(E)$ is an obvious multifractal analogue of the Hausdorff dimension $\operatorname{dim}(E)$ of $E$ whereas $\operatorname{Dim}_{\mu}^{q}(E)$ and $\Delta_{\mu}^{q}(E)$ are obvious multifractal analogues of the packing dimension $\operatorname{Dim}(E)$ and the prepacking dimension $\Delta(E)$ of $E$ respectively. In fact, it follows immediately from the definitions that $\operatorname{dim}(E)=\operatorname{dim}_{\mu}^{0}(E), \operatorname{Dim}(E)=\operatorname{Dim}_{\mu}^{0}(E)$ and $\Delta(E)=\Delta_{\mu}^{0}(E)$. Next we define multifractal dimension functions $\Lambda_{\mu}, B_{\mu}, b_{\mu}: \mathrm{R} \rightarrow[-\infty, \infty]$ by

$$
\begin{aligned}
\Lambda_{\mu}: q \rightarrow \Delta_{\mu}^{q}(\operatorname{supp} \mu), \\
\left.B_{\mu}: q \rightarrow \operatorname{Dim}_{\mu}^{q} \operatorname{supp} \mu\right), \\
b_{\mu}: q \rightarrow \operatorname{dim}_{\mu}^{q} q(\operatorname{supp} \mu) .
\end{aligned}
$$

One of the main importances of the multifractal measures $\mathscr{H}_{\mu}^{q, t}$ and $\mathscr{P}_{\mu}^{q, t}$, and the corresponding dimension functions $b_{\mu}, B_{\mu}$ and $\Lambda_{\mu}$ is due to the fact that the multifractal spectra functions $f_{\mu}$ and $F_{\mu}$ are bounded above by the Legendre transforms of $b_{\mu}$ and $B_{\mu}$, respectively, i.e.

$$
f_{\mu}(\alpha) \leq b_{\mu}^{*}(\alpha)
$$

and 


$$
F_{\mu}(\alpha) \leq B_{\mu}^{*}(\alpha)
$$

for all $\alpha \geq 0$, where the Legendre transform $f^{*}$ of a function $f: \mathbf{R} \rightarrow \mathbf{R}$ is defined by $f^{*}(x)=\inf _{y}(x y+f(y))$ (cf. [O11]). These inequalities may be viewed as rigorous versions of the Multifractal Formalism. Furhermore, for many natural families of measures we have $f_{\mu}(\alpha)=b_{\mu}^{*}(\alpha)$ and $F_{\mu}(\alpha)=B_{\mu}^{*}(\alpha)$ for all $\alpha \geq 0$, cf. [Co, Da1, Da2, O11, O12, O13]

It is clear by comparing the definitions of the measures $\mathscr{H}_{\mu}^{q, t}$ and $\mathscr{P}_{\mu}^{q, t}$, and definition (1.1) that $b_{\mu}(q)$ and $B_{\mu}(q)$ are mathematically rigorous versions of $\tau(q)$, and that the one-parameter families

$$
\left\{\mathscr{H}_{\mu}^{q, b_{\mu}(q)} \mid q \in \mathrm{R}\right\} \quad \text { and } \quad\left\{\mathscr{P}_{\mu}^{q, B_{\mu}(q)} \mid q \in \mathrm{R}\right\},
$$

play the role of the auxiliary measures $\left\{\mu_{q} \mid q \in \mathrm{R}\right\}$. In particular, we would expect that the measures $\left\{\mathscr{H}_{\mu}^{q, b_{\mu}(q)} \mid q \in \mathrm{R}\right\}$ and $\left\{\mathscr{P}_{\mu}^{q, B_{\mu}(q)} \mid q \in \mathrm{R}\right\}$ have properties similar to those of the auxiliary measures $\left\{\mu_{q} \mid q \in \mathrm{R}\right\}$. This has been proved rigorously for self-similar measures, for quasi self-similar measures and for self-conformal measures, cf. [Co, Da1, Da2, O11, O12, O'N]. In this paper we prove this result for arbitrary measures, cf. Theorem 1.1, Theorem 1.2, Theorem 2.1 and Theorem 2.2. As an application of Theorem 1.1 we obtain a formula relating the derivative of the multifractal box dimension function of $\mu$ and the entropy dimension of $\mu$, cf. Corollary 1.3.

For a function $f: \mathrm{R} \rightarrow \mathrm{R}$ and $x \in \mathrm{R}$, we denote the left and right derivative of $f$ at $x$ (if they exist) by $D_{-} f(x)$ and $D_{+} f(x)$, and we will denote the derivative of $f$ at $x$ (if it exists) by $f^{\prime}(x)$ or $D f(x)$.

We can now state two special cases of the main results. Theorem 1.1 should be viewed as a general version of (1.4) and states that if $B_{\mu}$ is differentiable at $q$, then the measure $\mathscr{H}_{\mu}^{q, b_{\mu}(q)}$ only sees the multifractal decomposition set $\left\{x \in \mathbf{R}^{d} \mid \lim _{r \backslash 0} \frac{\log \mu B(x, r)}{\log r}=-B_{\mu}^{\prime}(q)\right\}$. A more general result is obtained in Theorem 2.2.

THEOREM 1.1. Let $\mu$ be a Borel probability measure on $\mathrm{R}^{d}$ and $q \in \mathrm{R}$. Write $t=b_{\mu}(q)$ and $A_{ \pm}=D_{ \pm} B_{\mu}(q)$. If $b_{\mu}(q)=B_{\mu}(q)$, then

$$
-A_{+} \leq \liminf _{r \searrow 0} \frac{\log \mu B(x, r)}{\log r} \leq \limsup _{r \searrow 0} \frac{\log \mu B(x, r)}{\log r} \leq-A_{-} \quad \text { for } \mathscr{H}_{\mu}^{q, t} \text {-a.a. } x .
$$

Theorem 1.2 should be viewed as a general version of (1.5) and states that if $B_{\mu}$ is differentiable at $q$, then $\liminf _{r \backslash 0} \frac{\mathscr{H}_{\mu}^{q_{\mu}, b_{\mu}(q)}(B(x, r) \cap \operatorname{supp} \mu)}{\log r}=-q B_{\mu}^{\prime}(q)+$ $B_{\mu}(q)$ for $\mathscr{H}_{\mu}^{q, b_{\mu}(q)}$-a.a. $x$. A more general result is obtained in Theorem 2.1.

THEOREM 1.2. Let $\mu$ be a Borel probability measure on $\mathrm{R}^{d}$ and $q \in \mathrm{R}$. Write 
$t=b_{\mu}(q), T=B_{\mu}(q)$ and $A_{ \pm}=D_{ \pm} B_{\mu}(q)$. If $b_{\mu}$ is convex, $\mathscr{H}_{\mu}^{q, t}(\operatorname{supp} \mu)<\infty$ and $b_{\mu}(q)=B_{\mu}(q)$, then

$$
\begin{aligned}
\min \left(-q A_{-},-q A_{+}\right)+T & \leq \liminf _{r \searrow 0} \frac{\log \mathscr{H}_{\mu}^{q, t}(B(x, r) \cap \operatorname{supp} \mu)}{\log r} \\
& \leq \max \left(-q A_{-},-q A_{+}\right)+T, \\
\min \left(-q A_{-},-q A_{+}\right)+T & \leq \limsup _{r \searrow 0} \frac{\log \mathscr{H}_{\mu}^{q, t}(B(x, r) \cap \operatorname{supp} \mu)}{\log r}
\end{aligned}
$$

for $\mathscr{H}_{\mu}^{q, t}$-a.a. $x$.

By letting $q=1$ in Theorem 1.1 we obtain Corollary 1.3 relating the entropy dimension (or information dimension) of $\mu$ and the derivative of the upper multifractal box dimension function of $\mu$ at 1 . We will now define the multifractal box dimension function and the entropy dimension (or information dimension) of $\mu$. Let $\mu$ be a Borel probability measure on $\mathbf{R}^{d}$ and $q \in \mathrm{R}$. For $r>0$ write

$M_{\mu, r}^{q}=\sup \left\{\sum_{i} \mu\left(B\left(x_{i}, r\right)\right)^{q} \mid\left(B\left(x_{i}, r\right)\right)_{i \in \mathrm{N}}\right.$ is a centered packing of supp $\left.\mu\right\}$.

The lower and upper multifractal $q$-box dimensions (or $L^{q}$-dimensions) $\underline{\tau}_{\mu}(q)$ and $\bar{\tau}_{\mu}(q)$ of $\mu$ are defined by

$$
\underline{\tau}_{\mu}(q)=\liminf _{r \backslash 0} \frac{\log M_{\mu, r}^{q}}{-\log r}, \quad \bar{\tau}_{\mu}(q)=\limsup _{r \searrow 0} \frac{\log M_{\mu, r}^{q}}{-\log r} .
$$

If $\underline{\tau}_{\mu}(q)=\bar{\tau}_{\mu}(q)$ we refer to the common value as the $q$-box dimension of $\mu$ and denote it by $\tau_{\mu}(q)$. Next we define the entropy dimension (or information dimension) of $\mu$. For $r>0$ write

$$
\begin{aligned}
& h_{r}(\mu)=\left\{-\sum_{A \in \mathscr{A}} \mu(A) \log \mu(A) \mid\right. \\
& \left.\mathscr{A} \text { is a finite Borel partition of supp } \mu \text { with } \sup _{A \in \mathscr{A}} \operatorname{diam} A<r\right\} .
\end{aligned}
$$

The lower and upper entropy dimensions (or information dimensions) $\underline{D}(\mu)$ and $\bar{D}(\mu)$ of $\mu$ are defined by (cf. [Ré])

$$
\underline{D}(\mu)=\liminf _{r \searrow 0} \frac{h_{r}(\mu)}{-\log r}, \quad \bar{D}(\mu)=\limsup _{r \searrow 0} \frac{h_{r}(\mu)}{-\log r} .
$$

If $\underline{D}(\mu)$ and $\bar{D}(\mu)$ coincide, we write $D(\mu)$ for the common value. 
Since $b_{\mu}(1)=B_{\mu}(1)=0$ (cf. Proposition 4.1 below) and $\mathscr{H}_{\mu}^{1,0}$ is equivalent to $\mu$, Theorem 1.1 yields the following corollary by setting $q=1$.

COROLlaRy 1.3. Let $\mu$ be a Borel probability measure on $\mathrm{R}^{d}$.

(i) We have

$$
\begin{aligned}
-D_{+} B_{\mu}(1) & \leq \liminf _{r \searrow 0} \frac{\log \mu B(x, r)}{\log r} \leq \limsup _{r \searrow 0} \frac{\log \mu B(x, r)}{\log r} \\
& \leq-D_{-} B_{\mu}(1) \quad \text { for } \mu \text {-a.a. } x .
\end{aligned}
$$

If $B_{\mu}$ is differentiable at 1 , then (1.7) implies that $\lim _{r} \backslash 0 \frac{\log \mu B(x, r)}{\log r}=-B_{\mu}^{\prime}(1)$ for $\mu$-a.a. x, whence (cf. [Yo, Theorem 4.4])

$$
D(\mu)=-B_{\mu}^{\prime}(1) \text {. }
$$

(ii) Write $\tau=\bar{\tau}_{\mu}$. Since $-D_{+} \tau(1) \leq-D_{+} B_{\mu}(1)$ and $-D_{-} B_{\mu}(1) \leq-D_{-} \tau(1)$ (by Proposition 4.1 below), (1.7) implies that

$$
\begin{aligned}
-D_{+} \tau(1) & \leq \liminf _{r \searrow 0} \frac{\log \mu B(x, r)}{\log r} \leq \limsup _{r \searrow 0} \frac{\log \mu B(x, r)}{\log r} \\
& \leq-D_{-} \tau(1) \quad \text { for } \mu \text {-a.a. } x .
\end{aligned}
$$

If $\tau$ is differentiable at 1 , then (1.8) implies that $\lim _{r \backslash 0} \frac{\log \mu B(x, r)}{\log r}=-\tau^{\prime}(1)$ for $\mu$ a.a. $x$, whence (cf. [Yo, Theorem 4.4])

$$
D(\mu)=-\tau^{\prime}(1) .
$$

Inequality (1.8) has been suggested in numerous physics papers, cf. [HJKPS, HP], and heuristic arguments can be found in many recent physics textbooks on chaos and dynamical systems, see for example [BS, Section 11.3] or [Fe, (6.38)]. Inequality (1.8) has also been obtained independently by Heurteaux [He] and Ngai $[\mathrm{Ng}]$ using box counting arguments. Since (cf. Proposition 4.1 below) $-D_{+} \tau(1) \leq-D_{+} B_{\mu}(1)$ and $-D_{-} B_{\mu}(1) \leq-D_{-} \tau(1)$ where $\tau=\bar{\tau}_{\mu}$, we see that inequality (1.7) is sharper than Heurteaux's and Ngai's inequality (1.8). The next example shows that inequality (1.7) in general is strictly sharper than (1.8).

An Example. For $t \geq 7$ and $n=7,8,9, \ldots$ write $\varphi(t)=1+\frac{1}{\log (\log t)}$ and $a_{n}=n^{-\varphi(n)}$. We first observe that the series $\sum_{n=7}^{\infty} a_{n}$ is convergent. Indeed, there exists an integer $N \geq 7$ such that $\frac{\log n}{(\log (\log n))^{2}} \geq 2$ for all $n \geq N$, whence $\sum_{n=7}^{\infty} a_{n} \leq N+\sum_{n=N}^{\infty} \frac{1}{n \exp \left(\frac{\log n}{\log (\log n)}\right)} \leq N+\sum_{n=N}^{\infty} \frac{1}{n \exp (2 \log (\log n))}=N+$ $\sum_{n=N}^{\infty} \frac{1}{n(\log n)^{2}}<\infty$. Let $s=\sum_{n=7}^{\infty} a_{n} \in(0, \infty)$. Put $p_{n}=\frac{a_{n}}{s}$ and $s_{n}=\sum_{k=7}^{n} a_{k}$, and define the probability measure $\mu$ on $\mathrm{R}$ by 


$$
\mu=\sum_{n=7}^{\infty} p_{n} \delta_{s_{n}}
$$

Next we compute the multifractal box dimension functions, $\underline{\tau}_{\mu}$ and $\bar{\tau}_{\mu}$, and the multifractal packing dimension function, $B_{\mu}$, of $\mu$. We have

$$
\begin{gathered}
\underline{\tau}_{\mu}(q)=\bar{\tau}_{\mu}(q)=\tau_{\mu}(q)= \begin{cases}1-q & \text { for } 0 \leq q<1 ; \\
0 & \text { for } 1 \leq q,\end{cases} \\
B_{\mu}(q)=0 \text { for } 0 \leq q .
\end{gathered}
$$

Before proving (1.9) and (1.10), we consider some of the implications of these formulas. It follows from (1.9) that $D_{+} \tau_{\mu}(1)=0$ and $D_{-} \tau_{\mu}(1)=-1$, and inequality (1.8) therefore states that

$$
0 \leq \liminf _{r \searrow 0} \frac{\log \mu B(x, r)}{\log r} \leq \limsup _{r \searrow 0} \frac{\log \mu B(x, r)}{\log r} \leq 1 \quad \text { for } \mu \text {-a.a. } x .
$$

However, (1.10) shows that $D_{+} B_{\mu}(1)=D_{-} B_{\mu}(1)=0$, and inequality (1.7) therefore provides the following stronger statement,

$$
0 \leq \liminf _{r \searrow 0} \frac{\log \mu B(x, r)}{\log r} \leq \limsup _{r \searrow 0} \frac{\log \mu B(x, r)}{\log r} \leq 0 \quad \text { for } \mu \text {-a.a. } x,
$$

i.e.

$$
\lim _{r \searrow 0} \frac{\log \mu B(x, r)}{\log r}=0 \text { for } \mu \text {-a.a. } x .
$$

Inequality (1.7) is therefore in general strictly stronger than Heurteaux's and Ngai's inequality (1.8).

We will now prove (1.9) and (1.10).

Proof of (1.9). Fix $0 \leq q<1$ and choose $N \geq 14$ such that $\varphi(N / 2) q<1$. Next observe that $M_{\mu, r}^{q} \geq \sum_{k=7}^{n} p_{k}^{q} \geq s^{-q} \int_{n / 2}^{n} t^{-\varphi(n / 2) q} d t=c_{n} n^{1-\varphi(n / 2) q}$ for $n \geq N$ and $\frac{1}{2} a_{n+2} \leq r<\frac{1}{2} a_{n+1}$, where $\left(c_{n}\right)_{n}$ is a bounded sequence. Hence $\bar{\tau}_{\mu}(q) \geq \underline{\tau}_{\mu}(q) \geq \liminf _{n \rightarrow \infty} \frac{\log \left(c_{n} n^{1-\varphi(n / 2) q}\right)}{\log \left(\frac{1}{2}(n+2)^{-\varphi(n+2)}\right)}=1-q$. Since $\bar{\tau}_{\mu}$ is convex with $\underline{\tau}_{\mu}(0) \leq \bar{\tau}_{\mu}(0) \leq 1 \quad$ and $\quad \underline{\tau}_{\mu}(1)=\bar{\tau}_{\mu}(1)=0$, we now deduce that $\underline{\tau}_{\mu}(q)=\bar{\tau}_{\mu}(q)=1-q$ for $0 \leq q<1$. Next, let $1 \leq q$. Clearly $M_{\mu, r}^{q} \geq p_{7}^{q}$ for $0<r<\frac{1}{2} a_{8}$. This implies that $\bar{\tau}_{\mu}(q) \geq \underline{\tau}_{\mu}(q) \geq \liminf _{r \backslash 0} \frac{\log p_{7}^{q}}{-\log r}=0$. Since also $\underline{\tau}_{\mu}(q) \leq \bar{\tau}_{\mu}(q) \leq 0$ for $q \geq 1$, we now infer that $\underline{\tau}_{\mu}(q)=\bar{\tau}_{\mu}(q)=0$ for $q \geq 1$. This completes the proof of (1.9).

Proof of (1.10). Since $B_{\mu}$ is convex and decreasing (cf. [O11]) with 
$B_{\mu}(0)=\operatorname{Dim}(\operatorname{supp} \mu)=\operatorname{Dim}\left(\left\{s_{7}, s_{8}, s_{9}, \ldots, s\right\}\right)=0$ and $B_{\mu}(1)=0$ (cf. [O11]), we deduce that $B_{\mu}(q)=0$ for all $q \geq 0$. This completes the proof of (1.10).

Due to a formal analogy between equations (1.1) and (1.2) and the definitions of the so-called partition function and the free energy in thermodynamics, the quanteties $q$ and $\tau(q)$ are often in the physics literature interpreted as the inverse temperature and the free energy of "the physical system described by $\mu$ ". Similarly, due to a formal analogy between (1.5) and the socalled Gibbs ensemble in thermodynamics, $\mu_{q}$ is often interpreted as the most probable distribution of the system at inverse temperature $q$, i.e. the socalled "equilibrium state" of the system at inverse temperature $q$. The reader is referred to [BP, 128-132; BS, pp. 114-126; Ot, pp. 309-910] for a discussion of these and other analogies between multifractal analysis and thermodynamics. In thermodynamics, phase transitions are manifested as points of non-differentiability of the free energy $\tau(q)$ as a function of the inverse temperature $q$. Our study of the differentiability properties of the dimension functions $b_{\mu}$ and $B_{\mu}$ can therefore be interpreted as the study of "phase transitions" of the measure $\mu$, and the measures $\mathscr{H}_{\mu}^{p, b_{\mu}(p)}$ and $\mathscr{P}_{\mu}^{p, B_{\mu}(p)}$ can be regarded as natural "equilibrium states" of $\mu$ at "inverse temperature" $q$. It is natural to ask in what way the presence or absence of "phase transitions" of $\mu$ affect the smoothness of the "equilibrium states" of $\mu$ as a function of the "inverse temperature". In particular, we would expect that if the measure $\mu$ does not have a "phase transition" at $q$ (i.e. if the function $b_{\mu}$ is differentiable at $q$ ), then the "equilibrium states" of $\mu$ vary continuously with the "inverse temperature" at $q$. This question is formalised in Question 1.4 below. If $(X, \mathscr{E}, \lambda)$ is a measure space and $E \in \mathscr{E}$, then $\lambda\llcorner E$ denotes the restriction of $\lambda$ to $E$, i.e. $(\lambda\llcorner E)(F)=\lambda(E \cap F)$ for $F \in \mathscr{E}$.

Question 1.4. Let $\mu$ be a probability measure on $\mathrm{R}^{d}$ and $q \in \mathrm{R}$. Assume that for all $p$ in a neighbourhood of $q$ we have $b_{\mu}(p)=B_{\mu}(p)$ and $\mathscr{H}_{\mu}^{p, b_{\mu}(p)}(\operatorname{supp} \mu)<\infty$. Is the following result true? If $b_{\mu}$ is differentiable at $q$ (i.e. if $\mu$ does not have a "phase transition" at q), then the map

$$
p \rightarrow \mathscr{H}_{\mu}^{p, b_{\mu}(p)}\llcorner\operatorname{supp} \mu
$$

is continuous at $q$ with respect to the weak topology (i.e. the "equilibrium states" of $\mu$ vary continuously with the "inverse temperature" at q).

We now give a brief description of the organization the paper. Section 2 contains the statements of our main results. In Section 3 we state and prove various multifractal density theorems which will be used in the proofs of the main results, and in Section 4 we provide the proofs of the main results. 


\section{Statement of Results}

Let $\mathscr{P}\left(\mathrm{R}^{d}\right)$ denote the family of Borel probability measures on $\mathrm{R}^{d}$ and let $\mathscr{M}\left(\mathrm{R}^{d}\right)$ denote the family of Radon measures on $\mathrm{R}^{d}$. For a Radon measure $\mu$ on $\mathrm{R}^{d}$ we define the lower and upper local dimension of $\mu$ at $x$ by

$$
\bar{\alpha}_{\mu}(x)=\limsup _{r \searrow 0} \frac{\log \mu B(x, r)}{\log r}, \quad \underline{\alpha}_{\mu}(x)=\liminf _{r \searrow 0} \frac{\log \mu B(x, r)}{\log r} .
$$

If $\bar{\alpha}_{\mu}(x)$ and $\underline{\alpha}_{\mu}(x)$ agree we refer to the common value as the local dimension of $\mu$ at $x$ and denote it by $\alpha_{\mu}(x)$.

Our main results are formulated in terms of the Hausdorff dimension and the packing dimension of $\mu \in \mathscr{P}\left(\mathbf{R}^{d}\right)$. Intuitively the lower and upper Hausdorff (packing) dimension of $\mu$ equals the Hausdorff (packing) dimension of the "smallest" set with positive $\mu$-measure and the "smallest" set with full $\mu$ measure, respectively, and are thus natural measures of the singularity of $\mu$. The precise definitions are as follows. The lower Hausdorff dimension $\operatorname{dim}_{*} \mu$ of $\mu$ and the upper Hausdorff dimension $\operatorname{dim}^{*} \mu$ of $\mu$ are defined by

$$
\begin{aligned}
& \operatorname{dim}_{*} \mu=\inf \left\{\operatorname{dim} E \mid E \subseteq \mathrm{R}^{d} \text { and } \mu(E)>0\right\} \\
& \operatorname{dim}^{*} \mu=\inf \left\{\operatorname{dim} E \mid E \subseteq \mathrm{R}^{d} \text { and } \mu\left(\mathrm{R}^{d} \backslash E\right)=0\right\} .
\end{aligned}
$$

Similarly, the lower packing dimension $\operatorname{Dim}_{*} \mu$ of $\mu$ and the upper packing dimension $\operatorname{Dim}^{*} \mu$ of $\mu$ are defined by

$$
\begin{aligned}
& \operatorname{Dim}_{*} \mu=\inf \left\{\operatorname{Dim} E \mid E \subseteq \mathrm{R}^{d} \text { and } \mu(E)>0\right\} \\
& \operatorname{Dim}^{*} \mu=\inf \left\{\operatorname{Dim} E \mid E \subseteq \mathrm{R}^{d} \text { and } \mu\left(\mathrm{R}^{d} \backslash E\right)=0\right\} .
\end{aligned}
$$

Hausdorff and packings dimensions of a measure $\mu$ can also be expressed in terms of the local dimensions of $\mu$. Indeed, it follows from [HT] that

$$
\begin{array}{ll}
\operatorname{dim}_{*} \mu=\mu \text {-ess } \inf _{x} \underline{\alpha}_{\mu}(x), & \operatorname{dim}^{*} \mu=\mu \text {-ess } \sup _{x} \underline{\alpha}_{\mu}(x), \\
\operatorname{Dim}_{*} \mu=\mu \text {-ess } \inf _{x} \bar{\alpha}_{\mu}(x), & \operatorname{Dim}^{*} \mu=\mu \text {-ess } \sup _{x} \bar{\alpha}_{\mu}(x) .
\end{array}
$$

Recall that if $(X, \mathscr{E}, \lambda)$ is a measure space and $E \in \mathscr{E}$, then $\lambda\llcorner E$ denotes the restriction of $\lambda$ to $E$, i.e. $(\lambda\llcorner E)(F)=\lambda(E \cap F)$ for $F \in \mathscr{E}$. Also, recall that if $f: \mathrm{R} \rightarrow \mathrm{R}$ is a convex function and $x \in \mathrm{R}$, then we denote the left derivative and the right derivative of $f$ at $x$ by $D_{-} f(x)$ and $D_{+} f(x)$, respectively. We can now state our main reults. The proofs will be given in Section 4 .

Theorem 2.1. Let $\mu \in \mathscr{P}\left(\mathrm{R}^{d}\right)$ and $q \in \mathrm{R}$. Write $t=b_{\mu}(q), T=B_{\mu}(q), a_{ \pm}=$ $D_{ \pm} b_{\mu}(q)$ and $A_{ \pm}=D_{ \pm} B_{\mu}(q)$.

(i) If $\mathscr{H}_{\mu}^{q, t}(\operatorname{supp} \mu)<\infty$ and $b_{\mu}$ is convex, then 
$\left.\begin{array}{l}\text { If } q \leq 0 \text {, then }-q a_{-}+t \\ \text { If } 0 \leq q \text {, then }-q a_{+}+t\end{array}\right\} \leq \operatorname{Dim}_{*}\left(\mathscr{H}_{\mu}^{q, t}\llcorner\operatorname{supp} \mu) \leq \operatorname{Dim}^{*}\left(\mathscr{H}_{\mu}^{q, t}\llcorner\operatorname{supp} \mu)\right.\right.$ If in addition $b_{\mu}(q)=B_{\mu}(q)$, then

$\left.\begin{array}{l}\text { If } q \leq 0, \quad \text { then }-q A_{-}+T \\ \text { If } 0 \leq q, \quad \text { then }-q A_{+}+T\end{array}\right\} \leq \operatorname{dim}_{*}\left(\mathscr{H}_{\mu}^{q, t}\llcorner\operatorname{supp} \mu)\right.$ $\leq \operatorname{dim}^{*}\left(\mathscr{H}_{\mu}^{q, t}\llcorner\operatorname{supp} \mu) \leq\left\{\begin{array}{l}-q A_{+}+T \\ -q A_{-}+T\end{array}\right.\right.$

(ii) If $\mathscr{P}_{\mu}^{q, T}(\operatorname{supp} \mu)<\infty$, then

If $q \leq 0$, then
If $0 \leq q$, then $\operatorname{dim}_{*}\left(\mathscr{P}_{\mu}^{q, T}\llcorner\operatorname{supp} \mu) \leq \operatorname{dim}^{*}\left(\mathscr{P}_{\mu}^{q, T}\llcorner\operatorname{supp} \mu) \leq\left\{\begin{array}{l}-q A_{+}+T \\ -q A_{-}+T\end{array}\right.\right.\right.$

TheOREM 2.2. Let $\mu \in \mathscr{P}\left(\mathrm{R}^{d}\right)$ and $q \in \mathrm{R}$. Write $t=b_{\mu}(q)$ and $A_{ \pm}=$ $D_{ \pm} B_{\mu}(q)$. Assume that $b_{\mu}(q)=B_{\mu}(q)$. For $\mathscr{H}_{\mu}^{q, t}\llcorner\operatorname{supp} \mu$-a.a. $x$ we have

$$
-A_{+} \leq \underline{\alpha}_{\mu}(x) \leq \bar{\alpha}_{\mu}(x) \leq-A_{-} .
$$

Observe that Theorem 1.1 and Theorem 1.2 in the Introduction follows from Theorem 2.1 and Theorem 2.2. Figure 1 illustrates the geometrical significance of the numbers $-q a_{ \pm}+t$ and $-q A_{ \pm}+T$ in Theorem 2.1 and Theorem 2.2.
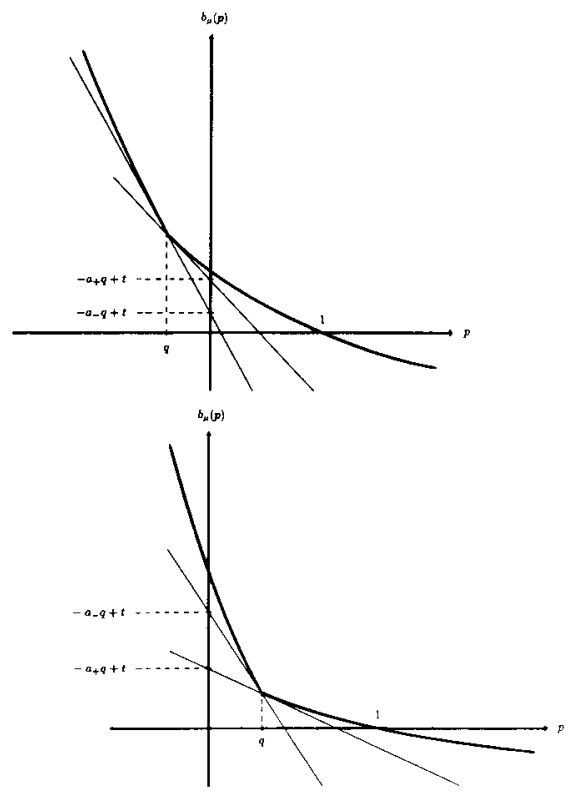
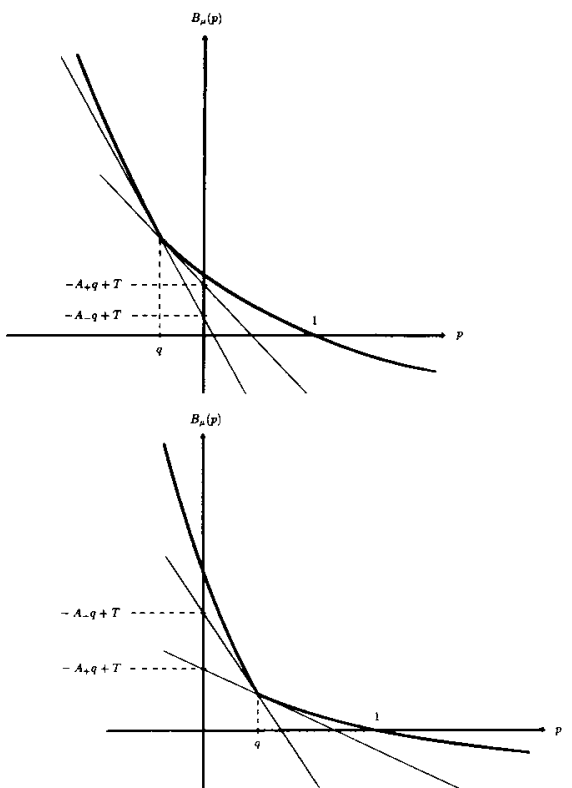

Fig. 1. The graphs of $b_{\mu}$ and $B_{\mu}$, and the geometrical significance of the numbers $-a_{ \pm} q+t$ and $-A_{ \pm} q+T$ for $q \leq 0$ and $0 \leq q$. 
Remarks. (1) It is natural to expect a rather subtle interplay between the local properties of the multifractal Hausdorff measure $\mathscr{H}_{\mu}^{q, t}$ and the multifractal packing measure $\mathscr{P}_{\mu}^{q, t}$ as indicated in Theorem 2.1. It follows from [O13] that there exist (self-affine) measures $\mu$ and $q \in \mathrm{R}$ such that $-q B_{\mu}^{\prime}(q)+$ $B_{\mu}(q)<0$. Hence, for such measures, the equality $\operatorname{Dim}^{*}\left(\mathscr{P}_{\mu}^{q, t}\llcorner\operatorname{supp} \mu)=\right.$ $\operatorname{Dim}_{*}\left(\mathscr{P}_{\mu}^{q, t}\llcorner\operatorname{supp} \mu)=-q B_{\mu}^{\prime}(q)+B_{\mu}(q)\right.$ cannot hold. It therefore seems necessary to introduce the regularity condition $b_{\mu}(q)=B_{\mu}(q)$, relating the local Hausdorff measure properties and the local packing measure properties.

(2) Taylor [Ta] defined a fractal to be any subset $E$ of a metric space which satisfies $\operatorname{dim} E=\operatorname{Dim} E$. For $q=0$ condition $b_{\mu}(q)=B_{\mu}(q)$ states that $\operatorname{dim}(\operatorname{supp} \mu)=b_{\mu}(0)=B_{\mu}(0)=\operatorname{Dim}(\operatorname{supp} \mu)$, i.e. supp $\mu$ satisfies Taylor's definition of a fractal. The regularity condition imposed on $\mu$ by requiring that $b_{\mu}(q)=B_{\mu}(q)$ can therefore be viewed as a multifractal version of Taylor's regularity condition.

(3) The numbers $A_{ \pm}=D_{ \pm} B_{\mu}(q)$ in Theorem 1.1 and Theorem 2.2 cannot be replaced by $a_{ \pm}=D_{ \pm} b_{\mu}(q)$. Examples in [O11] shows that there exist measures such that $-a_{-}<-a_{+}$. It is therefore natural to ask if the bounds in Theorem 2.2 are the best possible.

Question 2.3. Let $\mu \in \mathscr{P}\left(\mathrm{R}^{d}\right)$ and $q \in \mathrm{R}$. Write $t=b_{\mu}(q)$ and $A_{ \pm}=$ $D_{ \pm} B_{\mu}(q)$. Assume that $b_{\mu}(q)=B_{\mu}(q)$. Is

$$
\left(\mathscr{H}_{\mu}^{q, t}\llcorner\operatorname{supp} \mu) \text {-ess } \inf _{x} \underline{\alpha}_{\mu}(x)=-A_{+},\right.
$$

and

$$
\left(\mathscr{H}_{\mu}^{q, t}\llcorner\operatorname{supp} \mu) \text {-ess } \sup _{x} \bar{\alpha}_{\mu}(x)=-A_{-} ?\right.
$$

\section{Multifractal Density Theorems}

In this section we prove some multifractal density results which we will need in order to prove the main results; however, we believe that the density results below also have some interest in their own right.

Multifractal densities are defined as follows. Recall that $\mathscr{M}\left(\mathrm{R}^{d}\right)$ denotes the family of Radon measures on $\mathbf{R}^{d}$. Let $\mu, \nu \in \mathscr{M}\left(\mathbf{R}^{d}\right)$ and $q, t \in \mathbf{R}$. For $x \in \operatorname{supp} \mu$ we define the upper and lower $(q, t)$-density of $\nu$ at $x$ w.r.t. $\mu$ by

$$
\bar{d}_{\mu}^{q, t}(x, \nu)=\limsup _{r \searrow 0} \frac{\nu(B(x, r))}{\mu(B(x, r))^{q}(2 r)^{t}}, \quad \underline{d}_{\mu}^{q, t}(x, \nu)=\liminf _{r \backslash 0} \frac{\nu(B(x, r))}{\mu(B(x, r))^{q}(2 r)^{t}},
$$

cf. [O11].

Theorem 3.1 (Besicovitch's Covering Theorem). There exists a positive in- 
teger $\zeta$ such that the following holds: If $E \subseteq \mathrm{R}^{d}$ and $\left\{r_{x} \mid x \in E\right\}$ is a family of positive numbers satisfying $\sup _{x \in E} r_{x}<\infty$, then there exist $\zeta$ countable or finite subfamilies $\mathscr{V}_{1}, \ldots, \mathscr{V}_{\zeta}$ of $\left\{B\left(x, r_{x}\right) \mid x \in E\right\}$ such that

(1) For each $i=1, \ldots, \zeta$, the family $\mathscr{V}_{i}$ consists of pairwise disjoint sets.

(2) $E \subseteq \cup_{i} \cup_{B \in \mathscr{V}_{i}} B$.

Proof. See [deG, p. 5].

Theorem 3.2. Let $\mu, \nu \in \mathscr{M}\left(\mathrm{R}^{d}\right), q, t \in \mathrm{R}$ and let $E \subseteq$ supp $\mu$ be a Borel subset of supp $\mu$.

(i) If $\mathscr{H}_{\mu}^{q, t}(E)<\infty$ then

$$
\frac{1}{\zeta} \mathscr{H}_{\mu}^{q, t}(E) \inf _{x \in E} \bar{d}_{\mu}^{q, t}(x, \nu) \leq \nu(E) \leq \mathscr{H}_{\mu}^{q, t}(E) \sup _{x \in E} \bar{d}_{\mu}^{q, t}(x, \nu)
$$

where $\zeta$ is the constant that appears in Besicovitch's covering theorem.

(ii) If $\mathscr{P}_{\mu}^{q, t}(E)<\infty$ then

$$
\mathscr{P}_{\mu}^{q, t}(E) \inf _{x \in E} \underline{d}_{\mu}^{q, t}(x, \nu) \leq \nu(E) \leq \mathscr{P}_{\mu}^{q, t}(E) \sup _{x \in E} \underline{d}_{\mu}^{q, t}(x, \nu) .
$$

Proof.

(i) Proof of $\nu(E) \leq \mathscr{H}_{\mu}^{q, t}(E) \sup _{x \in E} \bar{d}_{\mu}^{q, t}(x, \nu)$. Follows from [O11, Theorem 2.14].

Proof of $\frac{1}{\zeta} \mathscr{H}_{\mu}^{q, t}(E) \inf _{x \in E} \bar{d}_{\mu}^{q, t}(x, \nu) \leq \nu(E)$. Let $a=\inf _{x \in E} \bar{d}_{\mu}^{q, t}(x, \nu)$. We may clearly assume that $a>0$. It suffices to prove that $\frac{1}{\zeta} \mathscr{H}_{\mu}^{q, t}(E)(a-\eta) \leq$ $\nu(E)+\varepsilon$ for all $\varepsilon>0$ and $0<\eta<a$. Now fix $\varepsilon>0$ and $0<\eta<a$. By inner regularity it suffices to prove that $\frac{1}{\zeta} \mathscr{H}_{\mu}^{q, t}(F)(a-\eta) \leq \nu(E)+\varepsilon$ for all closed subsets $F$ of $E$. Let $F$ be a closed subset of $E$. Since $\mathscr{H}_{\mu}^{q, t}(F)=$ $\sup _{H \subseteq F} \overline{\mathscr{H}}_{\mu}^{q, t}(H)$, it is sufficient to prove that

$$
\frac{1}{\zeta} \overline{\mathscr{H}}_{\mu}^{q, t}(H)(a-\eta) \leq \nu(E)+\varepsilon
$$

for all $H \subseteq F$. Now let $H \subseteq F$. For $r>0$ let $B(F, r)=\left\{x \in \mathrm{R}^{d} \mid \operatorname{dist}(F, x) \leq r\right\}$ denote the closed $r$-neighbourhood of $F$. Since $B(F, r) \searrow F$ as $r \searrow 0$ (because $F$ is closed) and $\overline{\mathscr{H}}_{\mu}^{q, t}(H) \leq \mathscr{H}_{\mu}^{q, t}(H)<\infty$ there exists $\delta>0$ such that

$$
\nu(B(F, \delta)) \leq \nu(F)+\frac{\varepsilon}{2 \zeta}, \quad \overline{\mathscr{H}}_{\mu}^{q, t}(H)-\frac{\varepsilon}{2(a-\eta)} \leq \overline{\mathscr{H}}_{\mu, \delta}^{q, t}(H) .
$$

Let $\mathscr{V}=\left\{B(x, r) \mid x \in H, 0<r<\delta, \nu(B(x, r)) \geq(a-\eta) \mu(B(x, r))^{q}(2 r)^{t}\right\}$. By assumption $H \subseteq \cup_{B \in \mathscr{V}} B$ and Besicovitch's covering theorem therefore implies that there exist $\zeta$ countable or finite subfamilies $\mathscr{V}_{1}, \ldots, \mathscr{V}_{\zeta}$ of $\mathscr{V}$ such that:

(1) for each $i=1, \ldots, \zeta$, the family $\mathscr{V}_{i}$ consists of pairwise disjoint sets;

(2) $H \subseteq \cup_{i} \cup_{B \in \mathscr{V}}$ B. 
Write $\mathscr{V}_{i}=\left(B\left(x_{i j}, r_{i j}\right)\right)_{j}$. We then have

$$
\begin{aligned}
\overline{\mathscr{H}}_{\mu}^{q, t}(H)(a-\eta) & \leq(a-\eta) \overline{\mathscr{H}}_{\mu, \delta}^{q, t}(H)+\frac{\varepsilon}{2} \\
& \leq(a-\eta) \sum_{i=1}^{\zeta} \sum_{j} \mu\left(B\left(x_{i j}, r_{i j}\right)\right)^{q}\left(2 r_{i j}\right)^{t}+\frac{\varepsilon}{2} \\
& \leq \sum_{i=1}^{\zeta} \nu\left(\cup_{j} B\left(x_{i j}, r_{i j}\right)\right)+\frac{\varepsilon}{2} \leq \zeta \nu(B(F, \delta))+\frac{\varepsilon}{2} \\
& \leq \zeta \nu(F)+\varepsilon \leq \zeta \nu(E)+\varepsilon .
\end{aligned}
$$

(ii) This statement is identical to [O11, Theorem 2.15].

TheOREM 3.3. Let $\mu \in \mathscr{M}\left(\mathrm{R}^{d}\right), q, t \in \mathrm{R}$ and let $E \subseteq$ supp $\mu$ be a measurable subset of supp $\mu$.

(i) If $\mathscr{H}_{\mu}^{q, t}(E)<\infty$ then

$$
1 \leq \bar{d}_{\mu}^{q, t}\left(x, \mathscr{H}_{\mu}^{q, t}\llcorner E) \leq \zeta \quad \text { for } \mathscr{H}_{\mu}^{q, t}\llcorner\operatorname{supp} \mu \text {-a.a. } x \in E\right.
$$

where $\zeta$ is the constant that appears in Besicovitch's covering theorem.

(ii) If $\mathscr{P}_{\mu}^{q, t}(E)<\infty$ then

$$
\underline{d}_{\mu}^{q, t}\left(x, \mathscr{P}_{\mu}^{q, t}\llcorner E)=1 \quad \text { for } \mathscr{P}_{\mu}^{q, t}\llcorner\operatorname{supp} \mu \text {-a.a. } x \in E .\right.
$$

Proof. (i) For $k \in \mathrm{N}$ write

$$
\begin{aligned}
& E_{k}^{-}=\left\{x \in E \mid \bar{d}_{\mu}^{q, t}\left(x, \mathscr{H}_{\mu}^{q, t}\llcorner E)<1-\frac{1}{k}\right\},\right. \\
& E_{k}^{+}=\left\{x \in E \mid \bar{d}_{\mu}^{q, t}\left(x, \mathscr{H}_{\mu}^{q, t}\llcorner E)>\zeta+\frac{1}{k}\right\} .\right.
\end{aligned}
$$

Put $\nu=\mathscr{H}_{\mu}^{q, t}\llcorner E$. For each $k \in \mathrm{N}$, Theorem 4.2 implies that

$$
\begin{aligned}
& \mathscr{H}_{\mu}^{q, t}\left(E_{k}^{-}\right)=\nu\left(E_{k}^{-}\right) \leq \mathscr{H}_{\mu}^{q, t}\left(E_{k}^{-}\right) \sup _{x \in E_{k}^{-}} \bar{d}_{\mu}^{q, t}(x, \nu) \leq \mathscr{H}_{\mu}^{q, t}\left(E_{k}^{-}\right)\left(1-\frac{1}{k}\right) \\
& \zeta \mathscr{H}_{\mu}^{q, t}\left(E_{k}^{+}\right)=\zeta \nu\left(E_{k}^{+}\right) \geq \mathscr{H}_{\mu}^{q, t}\left(E_{k}^{+}\right) \inf _{x \in E_{k}^{+}} \bar{d}_{\mu}^{q, t}(x, \nu) \geq \mathscr{H}_{\mu}^{q, t}\left(E_{k}^{+}\right)\left(\zeta+\frac{1}{k}\right),
\end{aligned}
$$

whence $\mathscr{H}_{\mu}^{q, t}\left(E_{k}^{-}\right)=0$ and $\mathscr{H}_{\mu}^{q, t}\left(E_{k}^{+}\right)=0$. Hence

$$
\mathscr{H}_{\mu}^{q, t}\left\{x \in E \mid \bar{d}_{\mu}^{q, t}\left(x, \mathscr{H}_{\mu}^{q, t}\llcorner E) \notin[1, \zeta]\right\}=\mathscr{H}_{\mu}^{q, t}\left(\left(\cup_{k} E_{k}^{-}\right) \cup\left(\cup_{k} E_{k}^{+}\right)\right)=0\right.
$$

which proves the desired result.

(ii) The proof of (ii) is similar to the proof of (i). 
REMARKS. (1) The results in Theorem 3.3 are multifractal generalizations of the density results in [Tr].

(2) Let $\mu, \nu \in \mathscr{M}\left(\mathrm{R}^{d}\right), q, t \in \mathrm{R}$ and $E$ be a Borel subset of supp $\mu$ such that $\mathscr{H}_{\mu}^{q, t}(E)<\infty$. If $\mu$ satisfies the doubling condition on $E$, i.e. if $\lim \sup _{r \searrow 0} \sup _{x \in E} \frac{\mu B(x, 2 r)}{\mu B(x, r)}<\infty$, then it follows from [O11] that the results in Theorem 3.2. (i) and Theorem 3.3. (i) can be strengthed: we have

$$
\mathscr{H}_{\mu}^{q, t}(E) \inf _{x \in E} \bar{d}_{\mu}^{q, t}(x, \nu) \leq \nu(E) \leq \mathscr{H}_{\mu}^{q, t}(E) \sup _{x \in E} \bar{d}_{\mu}^{q, t}(x, \nu)
$$

and

$$
\bar{d}_{\mu}^{q, t}\left(x, \mathscr{H}_{\mu}^{q, t}\llcorner E)=1 \quad \text { for } \mathscr{H}_{\mu}^{q, t}\llcorner\operatorname{supp} \mu \text {-a.a. } x \in E \text {. }\right.
$$

\section{Proofs of the Main Results}

In this section we prove the main results in the paper. We begin by stating a result about the behaviour of the dimension functions $b_{\mu}$ and $B_{\mu}$ which we will need later.

Proposition 4.1. Let $\mu \in \mathscr{P}\left(\mathrm{R}^{d}\right)$ and $q, t \in \mathrm{R}$.

(i) $\mathscr{P}_{\mu}^{q, t} \leq \overline{\mathscr{P}}_{\mu}^{q, t}$.

(ii) There exists an integer $\zeta$ such that $\mathscr{H}_{\mu}^{q, t} \leq \zeta \mathscr{P}_{\mu}^{q, t}$.

(iii) $\operatorname{dim}_{\mu}^{q} \leq \operatorname{Dim}_{\mu}^{q} \leq \Delta_{\mu}^{q}$, in particular $b_{\mu} \leq B_{\mu} \leq \Lambda_{\mu}$. Also $\Lambda_{\mu}=\bar{\tau}_{\mu}$.

(iv) $b_{\mu}$ is decreasing, and $B_{\mu}$ and $\bar{\tau}_{\mu}$ are convex and decreasing. Also $b_{\mu}(1)=B_{\mu}(1)=\bar{\tau}_{\mu}(1)=0$.

v) $D_{-} \bar{\tau}_{\mu}(1) \leq D_{-} B_{\mu}(1) \leq D_{+} B_{\mu}(1) \leq D_{+} \bar{\tau}_{\mu}(1)$.

Proof. Statements (i), (ii), (iii) and (iv) follow from [O11], and (v) follows immediately from (iii) and (iv).

We now turn to the proofs of the main results.

Proposition 4.2. Let $\mu \in \mathscr{P}\left(\mathrm{R}^{d}\right)$ and $q, t \in \mathrm{R}$.

(i) If $\mathscr{H}_{\mu}^{q, t}(\operatorname{supp} \mu)<\infty$, then we have for $\mathscr{H}_{\mu}^{q, t} \mathrm{~L}$ supp $\mu$-a.a. $x$,

$$
\left.\begin{array}{ll}
\text { If } q \leq 0, \text { then } & q \bar{\alpha}_{\mu}(x)+t \\
\text { If } 0 \leq q, \text { then } & q \underline{\alpha}_{\mu}(x)+t
\end{array}\right\} \leq \underline{\alpha}_{\mathscr{H}_{\mu}^{q, t}\llcorner\operatorname{supp} \mu}(x) \leq\left\{\begin{array}{l}
q \underline{\alpha}_{\mu}(x)+t \\
q \bar{\alpha}_{\mu}(x)+t
\end{array}\right.
$$

(ii) If $\mathscr{P}_{\mu}^{q, t}(\operatorname{supp} \mu)<\infty$, then we have for $\mathscr{P}_{\mu}^{q, t}\llcorner\operatorname{supp} \mu$-a.a. $x$, 


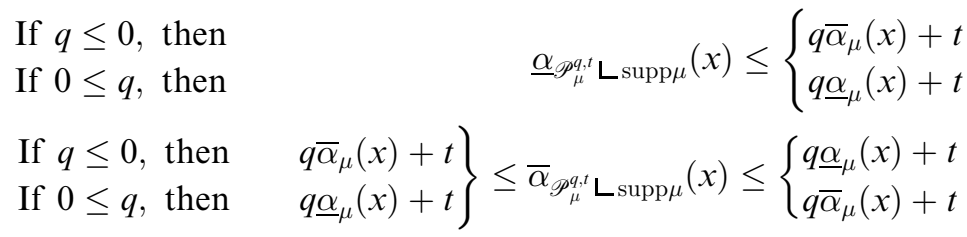

Proof. This result follows easily from Theorem 3.3 by taking logarithms.

Lemma 4.3. Let $\mu \in \mathscr{P}\left(\mathrm{R}^{d}\right)$. Fix $E \subseteq \operatorname{supp} \mu, q, p, t, s \in \mathrm{R}$ and $c>0$. Assume that for each $x \in E$ there exist positive numbers $r>0$ arbitrarily close to 0 such that

$$
\mu(B(x, r))^{q}(2 r)^{t} \leq c \mu(B(x, r))^{p}(2 r)^{s} .
$$

Then

$$
\mathscr{H}_{\mu}^{q, t}(E) \leq c \zeta \mathscr{P}_{\mu}^{p, s}(E)
$$

where $\zeta$ is the constant in Besicovitch's covering theorem.

Proof. Fix $F \subseteq E$ and $\delta>0$, and write $\mathscr{V}=\{B(x, r) \mid x \in F, 0<r<\delta$, $\left.\mu(B(x, r))^{q}(2 r)^{t} \leq c \mu(B(x, r))^{p}(2 r)^{s}\right\}$. By assumption $F \subseteq \cup_{B \in \mathscr{r}} B$, and it therefore follows from Besicovitch's covering theorem that there exist $\zeta$ countable or finite subfamilies $\mathscr{V}_{1}, \ldots, \mathscr{V}_{\zeta}$ of $\mathscr{V}$ such that:

(1) for each $i=1, \ldots, \zeta$, the family $\mathscr{V}_{i}$ consists of pairwise disjoint sets;

(2) $F \subseteq \cup_{i} \cup_{B \in \mathscr{V}_{i}} B$.

Write $\mathscr{V}_{i}=\left(B\left(x_{i j}, r_{i j}\right)\right)_{j}$. We clearly have

$$
\begin{aligned}
\overline{\mathscr{H}}_{\mu, \delta}^{q, t}(F) & \leq \sum_{i} \sum_{j} \mu\left(B\left(x_{i j}, r_{i j}\right)\right)^{q}\left(2 r_{i j}\right)^{t} \leq c \sum_{i} \sum_{j} \mu\left(B\left(x_{i j}, r_{i j}\right)\right)^{p}\left(2 r_{i j}\right)^{s} \\
& \leq c \sum_{i} \overline{\mathscr{P}}_{\mu, \delta}^{p, s}(F)=c \zeta \overline{\mathscr{P}}_{\mu, \delta}^{p, s}(F) .
\end{aligned}
$$

Letting $\delta \searrow 0$ now yields $\overline{\mathscr{H}}_{\mu}^{q, t}(F) \leq \zeta c \overline{\mathscr{P}}_{\mu}^{p, s}(F)$ for all $F \subseteq E$.

Hence, if $F \subseteq E$ and $E \subseteq \cup_{i} E_{i}$, then

$$
\begin{aligned}
\overline{\mathscr{H}}_{\mu}^{q, t}(F) & =\overline{\mathscr{H}}_{\mu}^{q, t}\left(\cup_{i}\left(F \cap E_{i}\right)\right) \leq \sum_{i} \overline{\mathscr{H}}_{\mu}^{q, t}\left(F \cap E_{i}\right) \\
& \leq c \zeta \sum_{i} \overline{\mathscr{P}}_{\mu}^{p, s}\left(F \cap E_{i}\right) \leq c \zeta \sum_{i} \overline{\mathscr{P}}_{\mu}^{p, s}\left(E_{i}\right) .
\end{aligned}
$$

It therefore follows that $\overline{\mathscr{H}}_{\mu}^{q, t}(F) \leq c \zeta \mathscr{P}_{\mu}^{p, s}(E)$ for all $F \subseteq E$, whence $\mathscr{H}_{\mu}^{q, t}(E) \leq c \zeta \mathscr{P}_{\mu}^{p, s}(E)$.

Proposition 4.4. Let $\mu \in \mathscr{P}\left(\mathrm{R}^{d}\right)$ and $q \in \mathrm{R}$.

(i) If $b_{\mu}$ is convex, then we have for $\mathscr{H}_{\mu}^{q, b_{\mu}(q)}\llcorner\operatorname{supp} \mu$-a.a. $x$, 


$$
-D_{+} b_{\mu}(q) \leq \bar{\alpha}_{\mu}(x), \quad \underline{\alpha}_{\mu}(x) \leq-D_{-} b_{\mu}(q) .
$$

(ii) We have for $\mathscr{P}_{\mu}^{q, B_{\mu}(q)}\llcorner\operatorname{supp} \mu$-a.a. $x$,

$$
-D_{+} B_{\mu}(q) \leq \bar{\alpha}_{\mu}(x), \quad \underline{\alpha}_{\mu}(x) \leq-D_{-} B_{\mu}(q) .
$$

Proof. (i) Proof of $-D_{+} b_{\mu}(q) \leq \bar{\alpha}_{\mu}(x)$ for $\mathscr{H}_{\mu}^{q, b_{\mu}(q)}\llcorner$ supp $\mu$-a.a. $x$. Write $a=D_{+} b_{\mu}(q)$. Fix $\varepsilon, \eta>0$ and let

$$
E=\left\{\begin{array}{l|l}
x \in \operatorname{supp} \mu \mid \liminf _{r \searrow 0} \frac{\mu B(x, r)}{(2 r)^{-a-\varepsilon}}>\eta
\end{array} .\right.
$$

It clearly suffices to prove that $\mathscr{H}_{\mu}^{q, b_{\mu}(q)}(E)=0$.

Since $b_{\mu}$ is convex, there exists $h>0$ such that $\frac{b_{\mu}(q+h)-b_{\mu}(q)}{h}<a+\varepsilon$, whence $b_{\mu}(q+h)<b_{\mu}(q)+h(a+\varepsilon)$, and we therefore deduce that

$$
\mathscr{H}_{\mu}^{q+h, b_{\mu}(q)+h(a+\varepsilon)}(\operatorname{supp} \mu)=0 .
$$

Also observe that for each $x \in E$ there exists a positive number $r_{x}$ such that

$$
\mu(B(x, r))^{q}(2 r)^{b_{\mu}(q)} \leq \eta^{-h} \mu(B(x, r))^{q+h}(2 r)^{b_{\mu}(q)+h(a+\varepsilon)} \quad \text { for } 0<r<r_{x} .
$$

It now follows easily from equations (4.1) and (4.2) that $\mathscr{H}_{\mu}^{q, b_{\mu}(q)}(E) \leq$ $\eta^{-h} \mathscr{H}_{\mu}^{q+h, b_{\mu}(q)+h(a+\varepsilon)}(E)=0$.

Proof of $\underline{\alpha}_{\mu}(x) \leq-D_{-} b_{\mu}(q)$ for $\mathscr{H}_{\mu}^{q, b_{\mu}(q)}\llcorner\operatorname{supp} \mu$-a.a. $x$. Write $a=$ $D_{-} b_{\mu}(q)$. Fix $\varepsilon, K>0$ and let

$$
E=\left\{\begin{array}{l|l}
x \in \operatorname{supp} \mu & \limsup _{r \searrow 0} \frac{\mu B(x, r)}{(2 r)^{-a+\varepsilon}}<K
\end{array}\right\} .
$$

It clearly suffices to prove that $\mathscr{H}_{\mu}^{q, b_{\mu}(q)}(E)=0$.

Since $b_{\mu}$ is convex, there exists $h>0$ such that $\frac{b_{\mu}(q)-b_{\mu}(q-h)}{h}>a-\varepsilon$, whence $b_{\mu}(q-h)<b_{\mu}(q)-h(a-\varepsilon)$, and we therefore deduce that

$$
\mathscr{H}_{\mu}^{q-h, b_{\mu}(q)-h(a-\varepsilon)}(\operatorname{supp} \mu)=0 .
$$

Next observe that for each $x \in E$ there exists a positive number $r_{x}$ such that

$$
\mu(B(x, r))^{q}(2 r)^{b_{\mu}(q)} \leq K^{h} \mu(B(x, r))^{q-h}(2 r)^{b_{\mu}(q)-h(a-\varepsilon)} \quad \text { for } 0<r<r_{x} .
$$

It now follows easily from equations (4.3) and (4.4) that $\mathscr{H}_{\mu}^{q, b_{\mu}(q)}(E) \leq$ $K^{h} \mathscr{H}_{\mu}^{q-h, b_{\mu}(q)-h(a-\varepsilon)}(E)=0$.

(ii) The proof of (ii) is similar to the proof of (i).

Proposition 4.5. Let $\mu \in \mathscr{P}\left(\mathrm{R}^{d}\right)$ and $q \in \mathrm{R}$. For $\mathscr{H}_{\mu}^{q, B_{\mu}(q)}$ L supp $\mu$-a.a. $x$ we have 


$$
-D_{+} B_{\mu}(q) \leq \underline{\alpha}_{\mu}(x) \leq \bar{\alpha}_{\mu}(x) \leq-D_{-} B_{\mu}(q) .
$$

Proof. Proof of $-D_{+} B_{\mu}(q) \leq \underline{\alpha}_{\mu}(x)$ for $\mathscr{H}_{\mu}^{q, B_{\mu}(q)}\llcorner\operatorname{supp} \mu$-a.a. $x$. Write $A=D_{+} B_{\mu}(q)$. Fix $\varepsilon, \eta>0$. It clearly suffices to prove that

$$
\mathscr{H}_{\mu}^{q, B_{\mu}(q)}\left(\left\{x \in \operatorname{supp} \mu \mid \limsup _{r \searrow 0} \frac{\mu B(x, r)}{(2 r)^{-A-\varepsilon}}>\eta\right\}\right)=0 .
$$

Since $B_{\mu}$ is convex (by Proposition 4.1), there exists $h>0$ such that $\frac{B_{\mu}(q+h)-B_{\mu}(q)}{h}<A+\varepsilon$, whence $B_{\mu}(q+h)<B_{\mu}(q)+h(A+\varepsilon)$, and we therefore deduce that

$$
\mathscr{P}_{\mu}^{q+h, B_{\mu}(q)+h(A+\varepsilon)}(\operatorname{supp} \mu)=0 .
$$

Let

$$
E=\left\{x \in \operatorname{supp} \mu \mid \limsup _{r \searrow 0} \frac{\mu B(x, r)}{(2 r)^{-A-\varepsilon}}>\eta\right\} .
$$

For each $x \in E$ there exist positive numbers $r$ arbitrarily close to 0 such that

$$
\mu(B(x, r))^{q}(2 r)^{B_{\mu}(q)} \leq \eta^{-h} \mu(B(x, r))^{q+h}(2 r)^{B_{\mu}(q)+h(A+\varepsilon)},
$$

and Lemma 4.3 and (4.5) therefore imply that $\mathscr{H}_{\mu}^{q, B_{\mu}(q)}(E) \leq$ $\eta^{-h} \zeta \mathscr{P}_{\mu}^{q+h, B_{\mu}(q)+h(A+\varepsilon)}(E)=0$.

Proof of $\bar{\alpha}_{\mu}(x) \leq-D_{-} B_{\mu}(q)$ for $\mathscr{H}_{\mu}^{q, B_{\mu}(q)}\llcorner$ supp $\mu$-a.a. $x$. Write $A=$ $D_{-} B_{\mu}(q)$. Fix $\varepsilon, K>0$. It clearly suffices to prove that

$$
\mathscr{H}_{\mu}^{q, B_{\mu}(q)}\left(\left\{x \in \operatorname{supp} \mu \mid \liminf _{r \searrow 0} \frac{\mu B(x, r)}{(2 r)^{-A+\varepsilon}}<K\right\}\right)=0 .
$$

Since $B_{\mu}$ is convex, there exists $h>0$ such that $\frac{B_{\mu}(q)-B_{\mu}(q-h)}{h}>A-\varepsilon$, whence $B_{\mu}(q-h)<B_{\mu}(q)-h(A-\varepsilon)$, and we therefore deduce that

$$
\mathscr{P}_{\mu}^{q-h, B_{\mu}(q)-h(A-\varepsilon)}(\operatorname{supp} \mu)=0 .
$$

Let

$$
E=\left\{x \in \operatorname{supp} \mu \mid \liminf _{r \searrow 0} \frac{\mu B(x, r)}{(2 r)^{-A+\varepsilon}}<K\right\} .
$$

For each $x \in E$ there exist positive numbers $r$ arbitrarily close to 0 such that

$$
\mu(B(x, r))^{q}(2 r)^{B_{\mu}(q)} \leq K^{h} \mu(B(x, r))^{q-h}(2 r)^{B_{\mu}(q)-h(A-\varepsilon)},
$$


and Lemma 4.3 and (4.6) therefore imply that $\mathscr{H}_{\mu}^{q, B_{\mu}(q)}(E) \leq$ $K^{h} \zeta \mathscr{P}_{\mu}^{q-h, B_{\mu}(q)-h(A-\varepsilon)}(E)=0$.

Proof of Theorem 2.1. Follows from Proposition 4.2, Proposition 4.4 and Proposition 4.5.

Proof of Theorem 2.2. Follows from Proposition 4.5.

\section{REFERENCES}

[AP] M. Arbeiter \& N. Patzschke, Random self-similar multifractals, Math. Nachr. 181 (1996), 5-42.

[BP] R. Badii \& A. Politi, Complexity. Hierarchical Structures and Scaling in Phusics, Cambridge Nonlinear Sci. Ser. 6, 1997.

[BS] C. Beck \& F. Schlögl, Thermodynamics of Chaotic Systems. An Introduction, Cambridge Nonlinear Sci. Ser. 4, 1993.

[CM] R. Cawley \& R. D. Mauldin, Multifractal decomposition of Moran fractals, Adv. in Math. 92 (1992), 196-236.

[Co] J. Cole, The Geometry of Graph Directed Self-Conformal Multifractals, Ph.D. Thesis, University of St. Andrews, 1998.

[Da1] M. Das, Packings and pseudo-packings: measures, dimensions and mutual singularity, preprint (1996).

[Da2] M. Das, Hausdorff measures, dimensions and mutual singularity, preprint(1996.

[deG] M. de Guzman, Differentiation of Integrals in $\mathrm{R}^{n}$, Lecture Notes in Math. 481, 1975.

[EM] G. A. Edgar \& R. D. Mauldin, Multifractal Decompositions of Digraph Recursive Fractals, Proc. London Math. Soc. 65 (1992), 604-628.

[Fa] K. J. Falconer, Fractal Geometry-Mathematical Foundations and Applications, John Wiley \& Sons, 1990.

[Fe] J. Feder, Fractals, Plenum, 1988.

[He] Y. Heurteaux, Estimations de la dimension inférieure et de la dimension supérieure des mesures, Ann. Inst. H. Poincaré Probab. Statist. 34 (1998), 309-338.

[HJKPS] T. C. Halsey, M. H. Jensen, L. P. Kadanoff, I. Procaccia \& B. J. Shraiman, Fractal measures and their singularities: The characterization of strange sets, Phys. Rev. A 33 (1986), 1141-1151.

[HP] H. Hentschel \& I. Procaccia, The infinite number of generalized dimensions of fractals and strange attractors, Physica 8D (1983), 435-444.

[HT] X. Hu \& S. J. Taylor, Fractal properties of products and projections of measures in $\mathrm{R}^{d}$, Math. Proc. Cambridge Philos. Soc. 115 (1994), 527-544.

[Ki] J. King, The singularity spectrum for general Sierpinski carpets, Adv. in Math. 116 (1995), 1-8.

[KG] J. King \& J. S. Geronimo, Singularity spectrum for recurrent IFS attractors, Nonlinearity 6 (1992), 337-348.

[Ma] P. Mattila, Geometry of Sets and Measures in Euclidean Spaces. Cambridge University Press, 1995.

$[\mathrm{Ng}] \quad$ S.-Z. Ngai, A dimension result arising from the $L^{q}$-spectrum of a measure, Proc. Amer. Math. Soc., 125 (1997), 2943-2951.

[O11] L. Olsen, A multifractal formalism, Adv. in Math. 116 (1995), 82-196.

[O12] L. Olsen, Random Geometrically Graph Directed Self-Similar Multifractals, Pitman Res. Notes Math. Ser. 307, 1994.

[O13] L. Olsen, Self-affine multifractal Sierpinski sponges in $\mathrm{R}^{d}$, Pacific J. Math. 183 (1998), $143-199$. 
[O’N] T. O'Neil, The multifratal spectrum of quasi self-similar measures, J. Math. Anal. Appl. 211 (1997), 233-257.

[Ot] E. Ott, Chaos in Dynamical Systems, Cambridge University Press, Cambridge, England, 1993.

[Pa] N. Patzschke, Self-conformal multifractals, Adv. in Appl. Math. 19 (1997), 486-513.

[Pes] Y. Pesin, Dimension type characteristics for invariant sets of dynamical systems, Russian Math. Surveys 43 (1988), 111-151.

[Pey] J. Peyriére, Multifractal measures, Proceedings of the NATO Advanced Study Institute on Probablistic ans Stochastic Methods in Analysis with Applications, Il Ciocco, pp. 175-186, NATO ASI Series, Series C: Mathematical and Physical Sciences, Vol 372, Kluwer Academic Press, Dordrecht, 1992.

[PW] Y. Pesin \& H. Weiss, A multifractal analysis of equilibrium measures for conformal expanding maps and Markov Moran geometric constructions, J. Statist. Phys. (1997), 233-275.

[Ra] D. Rand, The singularity spectrum $f(\alpha)$ for cookie-cutters, Ergodic Theory Dynamical Systems 9 (1989), 527-541.

[Ré] A. Rényi, Probability Theory, North-Holland, Amsterdam, 1970.

[RT] X. S. Raymond \& C. Tricot, Packing regularity of sets in n-space, Math. Proc. Cambridge Philos. Soc. 103 (1988), 133-145.

[RM] R. H. Riedi and B. Mandelbrot, Multifractal formalism for infinite multinomial measures, Adv. in Appl. Math. 16 (1995), 132-150.

[Ta] S. J. Taylor, A measure theory definition of fractals, Proceedings of the Measure Theory Conference at Oberwolfach. Supplemento Ai Rendiconti del Circolo Mathematico di Palermo, Ser. II, No 28 (1992), 371-378.

[TT] S. J. Taylor \& C. Tricot, Packing measure, and its evaluation for a Brownian path, Trans. Amer. Math. Soc. 288 (1985), 679-699.

[Tr] C. Tricot, Two definitions of fractional dimension, Math. Proc. Cambridge Philos. Soc. 91 (1982), 57-74.

[Yo] L.-S. Young, Dimension, entropy and Lyapunov Exponents, Ergodic Theory Dynamical Systems 2 (1982), 109-124.

DEPARTMENT OF MATHEMATICS

UNIVERSITY OF ST. ANDREWS

ST. ANDREWS

FIFE KY16 9SS

SCOTLAND

e-mail:1o@st-and.ac.uk 